\title{
EL ATLAS DE VENEZUELA A LA LUZ DE LA LEXICOGRAFÍA
}

\author{
Ana Isabel Navarro Carrasco \\ Universidad de Alicante
}

\section{RESUMEN}

El presente trabajo se ocupa de hacer un análisis de la obra El español de Venezuela de M. Alvar y confrontarla con el Diccionario Académico y los diccionarios de venezolanismos más representativos: Tejera 1993, y Núñez y Pérez 1998. Así se comprobará si hay en aquel Atlas voces que están recogidas o no por los diccionarios usuales y se corroborará o no la hipótesis de que los atlas lingüísticos están llenos de un caudal de léxico inmenso desconocido por la lexicografía.

Palabras clave: geografía lingüística, atlas lingüísticos, lexicografía, lexicología.

\section{ABSTRACT}

The aim of this paper is to analyse M. Alvar's El español de Venezuela and compare it to the Academic Dictionary and to two of the most outstanding dictionaries of Venezuelanisms, namely Tejera 1993, and Núñez and Pérez 1998. More specifically, we will examine whether the mentioned Atlas contains terms not included in general dictionaries, and will ultimately try to demonstrate the following hypothesis: linguistic atlases contain an immense wealth of lexical terms which is unknown to lexicographers.

Key Words: linguistic geography, linguistic atlases, lexicography, lexicology.

RECIBIDO: $19 / 02 / 2010$

APROBADO: 22/02/2011

El anunciado Atlas lingüistico de Hispanoamérica apareció bajo el título El español en América. En primer lugar, se publicó El español en el sur de los Estados Unidos (Alvar 2000a); continuó con El español en la República Dominicana (Alvar 2000b). Después, El español en Venezuela (Alvar 2001a) y, el último publicado, El español en Paraguay (Alvar 2001b). Aún la empresa no está concluida; esperamos El español en México, El español en Argentina y Uruguay, y El español en Chile.

En estos momentos nos vamos a ocupar de El español en Venezuela, y lo vamos a confrontar con el Diccionario Académico (Academia Española 2001; en adelante DRAE-01), y con el reciente Diccionario de ameri- 
canismos de la Asociación de Academias de la Lengua Española (López Morales 2010). Asimismo, consultamos los diccionarios de venezolanismos más representativos de los que disponemos hoy en día: Tejera 1993 y Núñez y Pérez 1998.

Durante mucho tiempo hemos trabajado en las líneas de geografía lingüística y lexicología y hemos concluido afirmando que los atlas lingüísticos están repletos de un léxico usual que no es recogido por los diccionarios ${ }^{1}$. Ahora vamos a analizar El español en Venezuela, para ver si esas voces están registradas o no por los diccionarios y en qué medida.

La pregunta es la siguiente: ¿el Diccionario de la Academia debe atender a los americanismos? La Academia, ya en su primer estatuto, afirma que «tiene por conveniente dar principio desde luego por la formación de un Diccionario de la lengua el más copioso que pudiera hacerse». Según se nos dice en la «Historia de la Real Academia» al principio del Diccionario de Autoridades, Academia Española 1726. Y en el Reglamento de la Real Academia Española se pretendía:

adquirir por diligencia de sus individuos de número y de los correspondientes, el mayor caudal posible de voces, locuciones y frases de uso particular en las provincias españolas y en los Estados hispanoamericanos, para emprender cuanto antes, y llevar a cabo, el más completo Diccionario de provincialismos que le sea dado publicar ${ }^{2}$.

La Academia, en la edición de 1925, cambió el nombre de Diccionario de la lengua castellana por Diccionario de la lengua española, precisamente por dar mayor cabida a voces de las distintas regiones de España e Hispanoamérica ${ }^{3}$.

Los diccionarios de venezolanismos, Tejera 1993 y Núñez y Pérez 1998 , incluyen lo propio pero no indican si eso que recogen es exclusivo de Venezuela o aparece también en otros países de Hispanoamérica.

Alvar 2001a se compone de tres tomos: el primero recoge «Palabras previas», I. Estudios, II. Encuestas y III. Textos, además de un índice de voces (pp. 1-350). El segundo comprende los mapas de tipo léxico (pp. 351-822), y el tercero los mapas de fonética, sintaxis y morfología (pp. 823-1316).

Después de las palabras previas, el primer tomo incluye los estudios, un total de ocho: «Venezuela, Norte, Sur, Este y Oeste» (pp. 23-29); «Español fronterizo: Venezuela-Colombia» (pp. 31-43); «Las palatales» (pp. 45-48); «Apostillas sociolingüísticas al habla de Falcón» (pp. 49-53); «Canarias y Venezuela» (pp. 55-79); «Análisis espectrográficos» (pp. 81-82);

\footnotetext{
1 Navarro Carrasco 1988, 1990, 1992, 1993, 1996, 1998, etcétera.

2 Academia Española 1861, capítulo I, artículo 2º, p. 2. Citado por Alvar 1982, p. 254 y nota 7 de la misma página.

${ }^{3}$ Academia Española 1925, p. II y XV de la «Advertencia» y Academia Española 1878.
} 
«Comentario estadístico de los sonogramas estudiados» por María Jesús Redondo (pp. 83-107); «Análisis espectrográficos de algunos sonidos venezolanos» por Josefa Dorta (pp. 109-117).

En las palabras precedentes se nos dice que el Atlas de Venezuela recoge información in situ de los hablantes en un momento determinado y que está realizado «con un método riguroso, que no tiene comparación con otros posibles» ${ }^{4}$. Alvar cuenta que en 1972 proyectó la realización de un Atlas de Hispanoamérica y, naturalmente, incluyó Venezuela, donde pensaba realizar cincuenta encuestas. En 1995 empezó por Valencia como punto de partida, donde el Dr. Navarro le ayudó en todo momento en las tareas relacionadas con la recogida de materiales, la búsqueda de informantes, la encuesta en sí y otras labores relacionadas con los trabajos de campo. En 1996 se encuestaron las regiones del oriente del país. En 1997 Manuel Alvar recorrió Venezuela de arriba abajo y de este a oeste realizando encuestas. En estas palabras iniciales, el Prof. Alvar agradece la generosidad de sus amigos venezolanos que le ayudaron organizando cursos en Caracas, en Mérida y Valencia, conferencias en cien Universidades, poniendo coches oficiales a su disposición y en resumen «todos, en todos los lugares, fueron dechados de generosidad» ${ }^{5}$.

Elena grabó todas las encuestas y registró las conversaciones libres, aparte de mil ayudas que surgen en las campañas de recogida de palabras.

Las encuestas fueron realizadas por Manuel Alvar. Cada día llevaba a cabo las casi 1.500 preguntas del cuestionario y por la noche transcribía las grabaciones que su mujer había hecho. Finalmente, el atlas se acabó con 49 puntos y 68 encuestas. En septiembre de 1998 se realizó la transcripción de materiales desde los cuadernos de campo a los de forma, con lo que la obra estaba preparada para la imprenta.

Para el acabado del Atlas, Florentino Paredes hizo los índices de palabras de los mapas y de los textos, así como la preparación de muchísimas informaciones. La elaboración de los mapas los ha llevado a cabo Teresa Alcázar. Manuel Alvar contó con los equipos de colaboradores de la Universidad de Alcalá, dirigidos por Antonio Alvar.

El creador del atlas da las gracias a todos cuantos han hecho posible esta obra: a los colegas del Instituto de Lingüística de Caracas, de las Universidades de Carabobo, de los Andes, de Eypolmetal Francisco de Misado (Coro), y de Oriente (Cumaná). Los trabajos de campo para realizar tan magna obra fueron patrocinados por dos ayudas de investigación del Ministerio de Educación y Cultura de España (a través del Instituto de España y del Consejo Superior de Investigaciones Científicas) y otras ayudas del Ministerio de Cultura y del Curso Superior de Filología. Igualmente, expresó su agradecimiento al Colegio Libre

\footnotetext{
${ }^{4}$ Alvar 2001a, p. 17.

${ }_{5}$ Alvar 2001a, p. 18.
} 
de Eméritos, que le ayudó a sufragar los gastos de los colaboradores, a la Agencia Española de Cooperación Internacional, a la Real Academia Española y a la Universidad de Venezuela.

Se realizaron encuestas en veinte Estados: Apure (4 localidades), Anzuátegui (2 localidades, pero en El Tigre se hicieron 2 encuestas), Barinas (4 localidades, pero en Arismendi 2 encuestas), Ciudad Bolívar (2 localidades), Carabobo (2 localidades, pero en Naguaragua 2 encuestas), Cojedes (1 localidad), Caracas (5 encuestas), Falcón (11 localidades, pero en Urumaco 2 encuestas), Guárico (4 localidades, pero en Las Mercedes del Llano 2 encuestas), Isla Margarita (1 localidad), Lara (2 localidades), Mérida (4 localidades, pero en Mérida 4 encuestas), Monagas (2 localidades), Portuguesa (2 localidades), Sucre (3 localidades), Trujillo (1 localidad), Táchira (2 localidades), Yaracuy (1 localidad, pero 2 encuestas), Zulia (4 localidades, pero en Maracaibo 3 encuestas).

Hay una relación de los informantes (pp. 123-126), donde aparece el nombre, la edad y la profesión de los mismos, además de algún que otro dato como, por ejemplo, los estudios, si es analfabeto, si viaja, etcétera.

El cuestionario por orden lógico comprende las pp. 127-137. Aparece una relación de los mapas desde el primero hasta el 931, el último. El cuestionario del léxico (mapas 1-454) incluye los siguientes campos:

I. El cuerpo humano.

II. Vestuario.

III. La casa. Cuestiones domésticas.

IV. Familia. Ciclo de la vida. La salud.

V. El mundo espiritual.

VI. Juegos y diversiones.

VII. Profesiones y oficios.

VIII. La enseñanza.

IX. El tiempo.

X. Accidentes topográficos.

XI. Agricultura.

XII. Animales y ganadería (insectos; aves, reptiles y peces; mamíferos; padres y crías; estado de los animales; conjunto de animales; vivienda de los animales; voces de los animales; voces para llamar a los animales; voces para espantar a los animales; partes del cuerpo de los animales, ordeño).

Después vienen las cuestiones de fonética (mapas 455-651), sintaxis (mapas 652-701) y morfología (mapas 702-931).

Hay una relación de las preguntas por orden alfabético (pp. 139148), de los signos fonéticos (pp. 149-154), de los signos accesorios (pp. 155-156).

La tercera parte del tomo I (pp. 157-265) está dedicada a los textos: un total de 49 en transcripción fonética y en ortografía normativa. Cada 
uno de ellos tiene un título. El primero se llama «Una pelea de gallos» y está contado por Francisco Javier Bezara de Achagua; el último lleva el nombre de «Miedo al Silbón» y está narrado por Enrique de Jesús Boves de la localidad de Dolores, Barinas.

Finalmente, el primer tomo incluye un «Índice de voces» (pp. 267346). Estos términos son las formas que aparecen en los mapas, ordenadas alfabéticamente. Es una parte muy importante de la obra, ya que en un momento dado se puede consultar dicha relación y comprobar rápidamente si una voz está o no incluida en el Atlas. Este índice de voces aparece, igualmente, en El español en el sur de los Estados Unidos y en El español en la República Dominicana. Hay que decir, no obstante, que todas las obras que constituyen la serie El español en América presentan la misma estructura y formato. Las palabras y expresiones que figuran en este índice alfabético están sujetas a unas normas de transcripción que se exponen previamente.

En cada tomo se ha incorporado la relación de puntos de encuesta, la descripción de signos fonéticos, los signos accesorios, las abreviaturas de otros atlas que se pueden relacionar con El español en Venezuela, así como los nombres de las localidades encuestadas con el fin de facilitar la consulta de los mapas. En cada carta lingüística hay una correspondencia con otros atlas. Se sabe que un mapa solo se puede comparar con otro si coincide la misma pregunta del cuestionario, si el nombre es el mismo. Cada página de los tomos dos y tres presenta la superficie de Venezuela cartografiada. Ahora bien, el estado de Falcón está desglosado de la carta general y aparece ampliado con el objeto de que puedan transcribirse con facilidad las respuestas obtenidas.

Después de analizar Alvar 2001a, hemos encontrado voces que están muy extendidas por Venezuela y que son desconocidas por el hablante del español peninsular. Son las siguientes:

1. Catire 'rubio' ${ }^{6}$ (II, 3$)^{7}$.

La voz está recogida por el DRAE-01 con localización en América. López Morales 2010 la sitúa en Co: N, E, Ve, Ec. También en Tejera 1993 y Núñez y Pérez 1998.

2. Capino 'albino' (II, 5$)^{8}$.

López Morales 2010 lo localiza en Ve. Recogido por Núñez y Pérez 1998.

${ }^{6}$ Fl 1, F 1, F 3, F 4b, F 7, F 10, Z 1, Z 2, Z 3, Z4, L 1, L 2, P 1, A 1, A 2, A 3, M 1a, b, d, M 2, B 4, Cr 1, S 2, Mo 1, Bo 1, Bo 2, N 1b, S 3. Ofrecemos las voces según su orden de aparición en los mapas para que guarden relación unas con otras. Por otro lado, indicamos la localidad para constatar que ciertamente la voz se usa allí. Si aparece el término en muchas localidades es porque hay constancia de que en cada una de ellas se ha estado y se ha comprobado su uso.

${ }^{7}$ El número romano indica el tomo y el arábigo el mapa.

${ }_{8} \mathrm{Z} \mathrm{1,} \mathrm{Z} \mathrm{2,} \mathrm{B} \mathrm{3,} \mathrm{A} \mathrm{1,} \mathrm{A} \mathrm{2,} \mathrm{A} \mathrm{3,} \mathrm{F} \mathrm{8,} \mathrm{M} \mathrm{1d,} \mathrm{M} \mathrm{2,} \mathrm{N} 1$ b. 
3. Picar 'guiñar el ojo' (II, 13) ${ }^{9}$.

Registrado por Tejera 1993 como «guiñar el ojo». Núñez y Pérez 1998 dicen que es «enamorar a alguien». López Morales 2010 da la misma definición que este último para RD y Ve.

4. Mocho 'cojo' (II, 53) ${ }^{10}$.

Tejera 1993 y Núñez y Pérez 1998 dan significados similares. López Morales 2010 con localización en CR, Pa, RD, PR, Co, Ve, Pe, Bo dice «persona o animal que carece de algún miembro o parte de él».

5. Cambeto 'zambo' (II, 54) $)^{11}$.

Recogido por el DRAE-01 con localización en Venezuela. Igualmente López Morales 2010. También en Tejera 1993 y Núñez y Pérez 1998.

6. Maneto 'estevado' (II, 55) ${ }^{12}$.

El DRAE-01 lo localiza en Honduras y Nicaragua. López Morales lo sitúa en Ve y Co, pero en este último país como poco usado. También aparece en Tejera 1993 y Núñez y Pérez 1998.

7. Flux 'traje [de hombre]' (II, 63) ${ }^{13}$.

El DRAE-01 lo da con localización en Andalucía y América. López Morales 2010 lo trae para Ni, Cu, RD, Ve y PR, este último como rural. También es recogido por Tejera 1993 y Núñez y Pérez 1998.

8. Paltó 'americana' (II, 64) ${ }^{14}$.

El DRAE-01 lo localiza en Venezuela. López Morales 2010 lo da para Ve y Ch. También aparece en Tejera 1993 y Núñez y Pérez 1998.

9. Braga 'mono (buzo, overol)' (II, 65) ${ }^{15}$.

López Morales 2010 lo sitúa en Ve. Registrado por Tejera 1993 y Núñez y Pérez 1998.

${ }^{9} \mathrm{Fl}$ 1, F 1, F 2, F 3, F 4, F 5, F 6, F 7, F 8, F 9, F 10, Z 1, Z 2, Z 3, Z 4, M 3, Ta 1, T 1, L 2, P 1, P 2, A 1, A 2, A 3, A 4, B 4, Cr 1, G 1, G 2, G 3, G 4, An 1, An 2, Bo 1, Bo 2, IM 1, Ca 1b, c, L 1, M 1c, d, M 2, Mo 1, N 1a, N 1b, S 1, S 3, Tá 2.

${ }^{10} \mathrm{~F}$ 1, F 2, F 4, F 5, F 6, F 7, F 8, F 9, F 10, Z 1, Z 2, L 1, L 2, T 1, M 3, Tá 1, L 2, P 1, P 2, A 1, A 2, A 3, Co 1, Cr 1, G 2, G 3, G 4, An 1, An 2, S 2, S 3, Mo 2, B 2, Ca 1b, M 1a, M 2, Mo 1, $\mathrm{N}$ la, $\mathrm{S} 1, \mathrm{Z} 3$.

${ }^{11}$ F 2, F 3, F 5, Z 1, Z 2, Z 3, Z 4, M 3, P 1, P 2, A 1, A 2, B 2, B 3, B 4, Cr 1, G 4, An 1, An 2, S 2, Bo 2, Ca 1b, c, Cr 1c, L 2, M 1a, c, d, M 2, N 1a, b, S 3.

${ }^{12}$ Fl 1, F 1, F 3, F 7, Co 1, B 2, B 4, A 4, G 1, G 2, S 2, An 2, Bo 2, Ca 1b, c, Cr 1b, d, M 1a.

${ }^{13} \mathrm{Fl} 1$, F 1, F 2, F 3, F 4, F 5, F 7, F 8, F 9, F 10, Z 1, Z 2, Z 3, Z 4, T 1, M 1, M 2, M 3, Tá 1, L 1, L 2, P 1, P 2, B 1, B 2, B 3, B 4, A 1, A 2, A 3, A 4, Cr 1, G 1, G 2, G 3, G 4, An 1, An 2, S 2, Mo 1, Mo 2, Bo 1, IM 1, Ca 1a, b, c, M 1a, b, c, d, N 1a, S 1, S 3.

${ }^{14} \mathrm{Fl}$ 1, F 1, F 2, F 3, F 4b, F 5, F 6, F 7, F 8, F 10, Z 1, Z 2, Z 4, L 1, L 2, T 1, P 1, M 1c, d, M 2, M 3, Tá 1, B 4, A 1, A 2, A 3, A 4, Cr 1, G 1, G 2, G 3, IM 1, S 2, S 3, An 2, Bo 1, Bo 2, Mo 1.

${ }^{15} \mathrm{Fl}$ 1, F 1, F 2, F 3, F 4, F 5, F 6, F 7, F 8, F 9, F 10, Z 1, Z 2, Z 3, Z 4, M 1a, M 1 b, c, d, M 2, M 3, Tá 1, T 1, L 1, L 2, P 1, P 2, B 1, B 2, B 3, B 4, Ca 1a, b, c, A 1, A 2, A 3, A 4, Cr 1, G 1, G 2, G 3, G 4, IM 1, An 1, An 2, S 2, Mo 1, Mo 2, Bo 1, Bo 2, N 1a, b, S 1, S 3, Tá 2. 
10. Yunta 'gemelos del puño de la camisa' (II, 67) ${ }^{16}$.

El DRAE-01 lo localiza en Puerto Rico y Venezuela. López Morales 2010 lo registra para Ve. También recogido por Tejera 1993 y Núñez y Pérez 1998.

11. Franela 'camiseta' (II, 68) ${ }^{17}$.

El DRAE-01 lo da para Venezuela. López Morales 2010 ofrece la siguiente área: Pa, RD, Co y Ve. También lo registran Tejera 1993 y Núñez y Pérez 1998.

12. Fondo 'enaguas' (II, 78) ${ }^{18}$.

El DRAE-01 lo registra en Venezuela. López Morales 2010 lo sitúa en Mx, RD, Co, Ve, Bo, además para Pe como rural. También en Tejera 1993 y Núñez y Pérez 1998.

13. Cintillo 'cinta para el pelo’ (II, 81) ${ }^{19}$.

López Morales 2010 ofrece la siguiente zona: Mx, Ho, Ni, Cu, Co: N, Ve, Pe, Bo, Ch, Py. Núñez y Pérez 1998 lo definen como «pieza estrecha hecha de diversos materiales en forma de semicírculo, que es utilizada por las mujeres para adornar o sujetar los cabellos».

14. Trenza 'cordones' (II, 85) ${ }^{20}$.

El DRAE-01 lo localiza en Venezuela. López Morales 2010 lo da para Ve, Bo: E, este último país como popular. También en Tejera 1993 y Núñez y Pérez 1998.

15. Pasador 'cerrojo' (II, 93) ${ }^{21}$.

No es registrado por los diccionarios.

16. Ponchera 'palangana' (II, 97) ${ }^{22}$.

El DRAE-01 da la localización de Venezuela. López Morales 2010 ofrece la siguiente área: RD, PR, Co: N, O, NE, Ve. También en Tejera 1993 y Núñez y Pérez 1998.

${ }^{16}$ Fl 1, F 1, F 3, F 4, F 6, F 7, F 10, Z 1, Z 3, Z 4, L 1, M 3, Tá 1, P 1, A 1, A 2, A 3, A 4, Co 1, B 2, B 4, Cr 1, G 1, G 2, S 2, S 3, Mo 2, An 2, Bo 1, Bo 2, Ca 1a, Ca 1b, c, Cr 1 e, N 1a, b.

${ }^{17} \mathrm{Fl} 1$ (franelilla), F 1, F 2, F 3 (franelilla), F 6, F 7, Z 1, Z 2 (franelilla), Z 4, Tá 1, T 1 (franelilla), A 1 (franelilla), A 2 (id.), P 1, P 2, G 2, G 4 (franelilla), IM 1 (id.), Mo 2 (id.), A 3 (id.), B 1 (id.), F 4b, G 3, L 1 (id.). M la (id.), M 1 b, c, d (id.), M 2 (id.), M 3a, b, N 1 a, Cr 1c (con media manga).

${ }_{18}$ Fl 1, F 1, F 2, F 3, F 4 b, F 5, F 6, F 7, F 8, F 9, F 10, Z 1, M 3, Tá 1, Tá 2, L 1, L 2, T 1, B 1, B 2, B 3, B 4, A 1, A 2, A3, A 4, P 1, P 2, G 1, G 2, G 3, IM 1, S 2, An 1, Mo 1, Mo 2, An 2, Bo 1 , Bo 2, Ca 1a, b, c, N 1a, b, S 1, S 3, Z 4 .

${ }^{19} \mathrm{Fl} \mathrm{1,} \mathrm{F} \mathrm{1,} \mathrm{F} \mathrm{2,} \mathrm{F} \mathrm{3,} \mathrm{F} \mathrm{4,} \mathrm{F} \mathrm{5,} \mathrm{F} \mathrm{6,} \mathrm{F} \mathrm{7,} \mathrm{F} \mathrm{8,} \mathrm{F} \mathrm{9,} \mathrm{F} \mathrm{10,} \mathrm{Z} \mathrm{1,} \mathrm{Z} \mathrm{2,} \mathrm{Z} \mathrm{4,} \mathrm{M} \mathrm{3,} \mathrm{Tá} \mathrm{1,} \mathrm{Tá} \mathrm{2,} \mathrm{L} \mathrm{1,} \mathrm{L} \mathrm{2,}$ T 1, A 1, A 2, A 3, A 4, P 1, Co 1, B 2, B 3, B 4, Cr 1, G 1, G 2, G 3, G 4, S 2, Mo 1, Mo 2, An 2, Bo 1, Bo 2, Ca la, b, c, M la, b, M 2, N 1a, S 1, S 3, Z 3.

${ }^{20} \mathrm{~F}$ 1, F 5, F 7, F 8, F 9, F 10, Z 1, M 3, A 2, A 3, P 1, P 2, Co 1, B 3, B 4, A 4, Cr 1, G 1, G 2, G 3, G 4, IM 1, S 2, An 1, An 2, Mo 2, Bo 1, Bo 2, A 1, Ca 1a, b, c, M 1 a, Mo 1, S 1, S 3.

${ }^{21}$ Fl 1, F 1, F 3, F 4 a, F 4b, F 6, F 7, F 8, F 10, Z 1, M 3, Tá 1, B 1, B 2, B 3, B 4, A 2, A 3, A 4, P 1, Co 1, Cr 1, G 1, G 2, G 3, IM 1, An 1, An 2, S 2, S 3, Mo 2, Bo 1, L 2, M 1 c, Mo 1.

${ }^{22}$ F 2, F 4a, F 6, F 8, Z 1, Z 2, Z 3, Z 4, M 3, T 1, P 1 (ponchero), B 2, B 3, Cr 1, G 2, G 3, G 4 , IM 1, An 1, Cr 1d, e, L 1, M la, b, c, d, M 2, N 1b, P 2, Tá 2 (ponchero). 
17. Cobija 'manta' (II, 105) ${ }^{23}$.

El DRAE-01 lo ofrece para América. López Morales 2010 registra una zona muy amplia: Mx, Gu, Ho, Py, ES, Ni, CR, Pa, Cu, RD, PR, Co, Ve, Ec, Pe, Bo, $\mathrm{Py}, \mathrm{Ar}, \mathrm{Ur}, \mathrm{Cu}, \mathrm{Ch}$, en este último país como poco usado. También es recogido por Tejera 1993 y Núñez y Pérez 1998.

18. Lavaplato(s) 'fregadero' (II, 123) ${ }^{24}$.

El DRAE-01 lo localiza en Bolivia, Chile, Colombia y Venezuela. López Morales 2010 lo trae para Ni, Co, Ve, Bo, Ch; para Py como popular. Recogido por Tejera 1993 y Núñez y Pérez 1998.

19. Morocho(s) 'gemelos o mellizos' (II, 140) ${ }^{25}$.

El DRAE-01 lo registra en Venezuela. Igualmente López Morales 2010. También aparece en Tejera 1993 y Núñez y Pérez 1998.

20. Pichirre 'tacaño' (II, 153) ${ }^{26}$.

El DRAE-01 lo localiza en Venezuela. López Morales 2010 lo trae para Ec y Ve. También en Tejera 1993 y Núñez y Pérez 1998.

21. A chuco, en chuco 'llevar a hombros' (II, 194) ${ }^{27}$ y 'llevar a cuestas' (II, $195)^{28}$.

No es recogido por el DRAE-01. Tampoco por López Morales 2010. Tejera 1993 , s. v. chuco, registra $a$ (en) chuco «a horcajadas sobre los hombros de una persona» para el Estado de Lara.

22. Papagayo 'cometa' (II, 204) ${ }^{29}$.

El DRAE-01 lo localiza en Venezuela. López Morales 2010 lo sitúa en Mx: SE, Ve y Bo: O. También lo recogen Tejera 1993 y Núñez y Pérez 1998.

23. Volantín 'cometa' (II, 204) ${ }^{30}$.

El DRAE-01 da la siguiente localización: oeste de Argentina, Chile, Cuba, Puerto Rico y Venezuela. López Morales 2010 ofrece la zona de Mx, Cu: E,

${ }^{23}$ Fl 1, F 1, F 2, F 3, F 4, F 6, F 7, F 8, Z 1, Z 2, Z 4, M 3, Tá 1, L 1, T 1, B 1, B 2, B 4, A 1, A 2, A 3, A 4, P 1, P 2, Co 1, Cr 1, G 1, G 2, G 3, IM 1, An 1, An 2, S 2, Mo 2, Bo 1, Bo 2, Ca 1a, b, c, L 2, M 1 a, b, c, d, M 2, Mo 1, N 1 a, b, S 1, S 3, Tá 2, Z 3.

${ }^{24}$ F 1, F 2, F 4a, F 4b, F 5, F 7, Tá 1, Z 2, T 1, P 2, B 3, A 1, Co 1, G 1, G 3, IM 1, A 2, B 2, Ca 1a, b, c, Cr 1d, e, L 1, M 1d, M 2, Mo 1, Tá 2.

${ }^{25} \mathrm{Fl} \mathrm{1,} \mathrm{F} \mathrm{1,} \mathrm{F} \mathrm{2,} \mathrm{F} \mathrm{3,} \mathrm{F} \mathrm{4,} \mathrm{F} \mathrm{5,} \mathrm{F} \mathrm{6,} \mathrm{F} \mathrm{7,} \mathrm{F} \mathrm{8,} \mathrm{F} \mathrm{9,} \mathrm{F} \mathrm{10,} \mathrm{Z} \mathrm{1,} \mathrm{Z} \mathrm{2,} \mathrm{Z} \mathrm{4,} \mathrm{M} \mathrm{3,} \mathrm{Tá} \mathrm{1,} \mathrm{T} \mathrm{1,} \mathrm{B} \mathrm{1,} \mathrm{B} \mathrm{2,}$ B 3, B 4, A 1, A 2, A 3, A 4, L 2, P 1, P 2, Co 1, Cr 1, G 1, G 2, G 3, G 4, IM 1, S 2, S 3, An 1, An 2, Mo 2, Bo 1, Ca 1a, b, Ca 1c, L 1, M 1a, b, c, M 2, Mo 1, N 1a, b, S 1, Z 3.

${ }^{26}$ F 4b, F 5, F 6, F 7, F 9, Z 1, Z 4, B 1, B 4, A 4, G 1, G 4, An 2, Bo 1, Ca 1c, Mo 1, N 1a, b, S 3, Z 3 .

${ }^{27}$ F 1, F 4, F 5, F 6, F 7, F 8, F 10, M 3, A 1, A 3, P 1, P 2, Co 1, Ca 1 a, b, c, N 1a, b.

${ }^{28} \mathrm{Fl} 1, \mathrm{~F} 4, \mathrm{~F} 5, \mathrm{P} 2, \mathrm{~B} 2, \mathrm{~N} 1 \mathrm{a}$.

${ }_{29}$ F 1, F 6, Z 1, P 2, A 1, A 2, A 4, B 1, B 2, B 3, B 4, Cr 1, G 2, G 3, G 4, IM 1, An 1, An 2, S 1 , S 2, S 3, Bo 1, Bo 2, Ca 1 a, b, c, Co 1, L 1, L 2, M 1 a, b, c, d, M 3 a, b, N 1 a, b, Z 4.

${ }^{30} \mathrm{Fl} \mathrm{1,} \mathrm{F} \mathrm{2,} \mathrm{F} \mathrm{3,} \mathrm{F} 4$ a, b, F 5, F 6, F 7, F 8, F 9, F 10, Z 1, T 1, B 3, Z 2, Z 3, Z 4. 
PR, Ve: O, Ch, Ar: O, No, Bo, en este último país como poco usado. También lo recogen Tejera 1993 y Núñez y Pérez 1998.

24. Limosnero 'mendigo' (II, 208) ${ }^{31}$.

El DRAE-01 indica que se usa más en Andalucía y América. Según López Morales 2010 figura en la siguiente área: Ni, CR, Pa, Cu, RD, Co, Ve, Ec, Pe, Bo, Ch, Py, Ar. También es recogido por Tejera 1993 y Núñez y Pérez 1998.

25. Ganchos 'horquillas para el pelo' (II, 217) ${ }^{32}$.

El DRAE-01 lo recoge con localización en América. López Morales 2010 lo da para EU, Gu, Ho, ES, Ni, CR, Pa, Cu, RD, Co, Ve, Pe, Bo, Py, Ar; y para Ch como obsolescente. También aparece en Tejera 1993 y Núñez y Pérez 1998.

26. Kinder 'enseñanza anterior a la primaria' (II, 221) ${ }^{33}$.

El DRAE-01 no lo registra. López Morales 2010 lo sitúa en EU, Mx, Gu, Ho, $\mathrm{Pa}, \mathrm{RD}, \mathrm{PR}, \mathrm{Co}, \mathrm{Ve}, \mathrm{Ec}, \mathrm{Bo}, \mathrm{Ch}$, Ar. Tejera 1993 ofrece kindergarterina con el significado de «maestra de kindergarten o jardín de infancia». Núñez y Pérez 1998 dicen de kinder lo siguiente: «instituto educativo para niños de corta edad».

27. Bulto 'cartera' (II, 222) $)^{34}$.

El DRAE-01 lo localiza en Costa Rica y Venezuela. López Morales 2010 lo registra para Ni, RD, PR, Ve, Bo; y para CR como poco usado. También aparece en Tejera 1993 y Núñez y Pérez 1998.

28. Raspar 'suspender, desaprobar' (II, 229) ${ }^{35}$.

El DRAE-01 lo da para Venezuela. Igualmente López Morales 2010. También lo recogen Tejera 1993 y Núñez y Pérez 1998.

29. Jojoto 'elote' (mazorca tierna)' (II, 304) ${ }^{36}$.

El DRAE-01 lo registra en Venezuela. López Morales 2010 lo trae para Co: E y Ve. También en Tejera 1993 y Núñez y Pérez 1998.

${ }^{31} \mathrm{Fl} \mathrm{1,} \mathrm{F1,} \mathrm{F} \mathrm{2,} \mathrm{F} \mathrm{3,} \mathrm{F} 4$ a, b, F 5, F 6, F 7, F 8, F 9 F 10, Z 2, T 1, B 1, B 2, B 3, B 4, P 1, P 2, A 3, A 4, Co 1, Cr 1, G 1, G 2, G 3, G 4, IM 1, S 2, An 1, An 2, Mo 2, Bo 1, Bo 2, Ca 1 a, b, Ca 1c, Cr 1d, L 1, L 2, M 1 a, b, d, M 2, M 3 a, Mo 1, N 1 a, b, S 3, Tá 1, Tá 2, Z 3, Z 4.

${ }_{32}$ Fl 1, F 1, F 2, F 3, F 4, F 5, F 6, F 7, F 8, F 9, F 10, Z 1, Z 2, Z 4, M 3, Tá 1, T 1, B 3, A 1, A 2, A 3, A 4, L 2, P 1, P 2, Co 1, B 4, Cr 1, G 1, G 2, IM 1 (ganchillo), An 1, An 2, S 2, Bo 1, Bo 2, Ca 1 b, c, L 1, M 1 a, b, c, d, M 2, Mo 1, N 1 a, b, S 1, S 3, Tá 2, Z 3.

${ }_{33}$ F 1, F 2, F 3, F 4, F 4b, F 7, F 8, Z 1, Z 2, Z 4, L 2, P 2, Co 1, A 4, G 1, G 2, G 3, G 4, IM 1, An 1 , S 2, B 1 (kindergarten), B 2, B 3, Ca 1a, b, c, Cr 1e, L 1 (kindergarten), M 1a, b, c, d, N 1a, S 1, Tá 2.

${ }_{34} \mathrm{Fl} \mathrm{1,} \mathrm{F} \mathrm{1,} \mathrm{F} \mathrm{2,} \mathrm{F} \mathrm{3,} \mathrm{F} \mathrm{4,} \mathrm{F} \mathrm{5,} \mathrm{F} \mathrm{6,} \mathrm{F} \mathrm{7,} \mathrm{F} \mathrm{10,} \mathrm{Z} \mathrm{1,} \mathrm{Z} \mathrm{2,} \mathrm{Z} \mathrm{4,} \mathrm{M} \mathrm{3,} \mathrm{L} \mathrm{1,} \mathrm{L} \mathrm{2,} \mathrm{T} \mathrm{1,} \mathrm{B} \mathrm{1,} \mathrm{A} \mathrm{1,} \mathrm{A} \mathrm{2,}$ A 3, A 4, P 1, P 2, Cr 1, G 2, G 3, An 1, S 2, Mo 2, Bo 1, Bo 2, Cr 1 e, N 1a, b, Tá 2.

${ }_{35}$ Fl 1, F 1, F 3, F 7, F 8, F 10, Z 1, Z 3, Z 4, L 1, T 1, B 1, B 2, B 4, A 1, A 2, A 3, A 4, B 4, Cr 1, G 4, IM 1, Mo 2, Ca 1b, c, F 4b, L 2, M1a, b, M 1c, M 1d, M 3a, M 3b, Mo 1, N 1a, N 1b, P 1, S 1, Tá 2 .

${ }^{36} \mathrm{Fl}$ 1, F 1, F 2, F 3, F 4, F 5 (kojoto), F 6 (id.), F 7, F 8 (kojoto), F 9 (id.), F 10 (id.), Z 1, Z 2, Z 3 , Z 4, Tá 1, L 1, T 1, B 1, B 4, A 1, A 2, A 3, A 4, P 1, P 2, Co 1, Cr 1, G 1, G 2, G 3, G 4, An 1, An 2, S 2, Mo 2, Bo 1, Bo 2, B 2, B 3, Ca 1a, Ca 1b, Ca 1c, Cr 1 e, L 2, M 1a, b, c, d, M 2, M 3b, N 1a, b, S 3, Tá 2 . 
30. Cambur 'plátano, banano (planta)' (II, 307) ${ }^{37}$.

El DRAE-01 no ofrece localización. Lo da como voz general. López Morales 2010 la trae para Ve. Figura en Tejera 1993 y Núñez y Pérez 1998.

31. Cambural 'platanar (terreno de plátanos)' $(\mathrm{II}, 308)^{38}$.

El DRAE-01 lo localiza en Venezuela. Igualmente López Morales 2010. También en Tejera 1993 y Núñez y Pérez 1998.

32. Mecate 'ronzal' (II, 316) ${ }^{39}$.

El DRAE-01 lo recoge para América Central, México y Venezuela. López Morales 2010 ofrece la siguiente zona: Mx, Gu, Ho, ES, Ni, CR, Pa, Co: E, O, Ve; en Ec poco usado. También lo traen Tejera 1993 y Núñez y Pérez 1998.

33. Halar 'arrastrar' (II, 319) ${ }^{40}$.

El DRAE-01 con localización en Venezuela dice que es «arrastrar por la fuerza». También lo registran Tejera 1993 y Núñez y Pérez 1998. Las definiciones de López Morales 2010 no concuerdan con nuestro mapa.

34. Mata de limón 'limonero' (II, 320) ${ }^{41}$, mata de higo 'higuera' (II, 322) ${ }^{42}$, mata, mata de naranja, mata naranjo 'naranjo' (II, 325) ${ }^{43}$, mata de manzana, mata de manzano, mata 'manzano' (II, 326) ${ }^{44}$, mata de pera 'pe-

${ }_{37}$ Fl 1, F 1, F 2, F 3, F 4, F 5, F 6, F 7, F 8, F 9, F 10, Z 1, Z 2, Tá 1, L 1, L 2, T 1, B 1, B 3, B 4, A 2, A 3, A 4, P 1, P 2, Co 1, Cr 1, G 1, G 2, G 3, G 4, IM 1, An 1, An 2, S 2, Mo 2, Bo 1, B 2, Ca 1a, Ca 1b, Ca 1c, M 1a, M 1b, c, d, M 2, M 3a, b, Mo 1, N 1a, b, S 1, S 3, Tá 2.

${ }^{38} \mathrm{Fl}$ 1, F 1, F 3, F 4, F 5, F 6, F 7, F 8, F 9, F 10, Z 1, M 3, T 1, L 1, L 2, P 2, B 1, B 2, B 3 , B 4, A 3, A 4, Co 1, Cr 1, G 1, G 2, G 3, IM 1, S 1, S 2, S 3, An 1, An 2, Mo 1, Mo 2, Bo 1, Bo 2, Ca 1 a, b, c, Cr 1 e, M 1 a, b, c, d, M 2, N 1 a, b, Tá 2 .

${ }^{39}$ Fl 1, F 2, F 3, Tá 1, B 1, P 1, P 2, Co 1, B 4, Cr 1, G 1, Bo 2, N 1 a, Tá 2.

${ }^{40} \mathrm{Fl}$ 1, F 1, F 2, F 3, F 4, F 4b, F 5, F 7, F 9, F 10, Z 1, Z 2, Z 3, Z 4, Tá 1, T 1, B 1, B 2, B 3, A 2, A 3, A 4, L 2, P 1, P 2, Co 1, B 4, Cr 1, G 1, G 2, G 4, An 1, An 2, S 2, Mo 2, Bo 1, Bo 2, Ca 1a, b, c, L 1, M 1 a, b, d, M 1c, M 2, M 3a, M 3b, Mo 1, N 1a, S 1, S 3, Tá 2.

${ }^{41} \mathrm{Fl}$ 1, F 1, F 2, F 3, F 4, F 4b, F 5, F 6, F 7, F 8, F 9, F 10, Z 1, Z 2, Z 3, Z 4, Tá 1, T 1, B 1, B 2, B 3, B 4, A 1, A 2, A 3, Co 1, Cr 1, Cr 1d, e, G 1, G 2, G 3, IM 1, An 1 (mata), An 2 (id.), S 2 (id.), Mo 2, Bo 1, Bo 2, Ca 1a, c, C 1b, M 1a, d, M 2, Mo 1, N 1 b, P 1, P 2, S 1.

${ }^{42} \mathrm{Fl} 1, \mathrm{~F} 1$, F 2, F 3, F 4 a, F 4b, F 5 (mata), F 6 (id.), F 7, Z 1, Z 2, Z 4, Tá 1, T 1, P 1, P 2, B 1, B 2, B 3 B 4 (mata), A 1, A 2, A 3, Cr 1, G 1 (mata), G 2 (id.), IM 1, S 1 (mata), S 2, S 3, An 1, An 2, Mo 2, Bo 1 (mata), Ca 1 a, b, c, L 2, M 1a, c, d, M 1 b, Mo 1 (mata), N 1 b.

${ }^{43}$ Cuando no indicamos nada se trata de mata de naranja: Fl 1, F 2 (mata naranjo), F 4, F 5 (mata), F 6 (id.), F 8 (id.), F 9 (id.), Z 1 (id.), Z 1c, Z 2 (mata naranjo), Z 4 (mata), Tá 1 (id.), T 1 (id.), A 2, A 3, P 1 (mata), P 2 (id.), B 3, Co 1 (mata), Cr 1, G 2 (mata), G 3 (id.), IM 1, Mo 2, B 2 (mata), Ca 1 a, b, c, F 3 (mata de naranjo), M 1d, M 2, Mo 1 (mata), N 1b, $\mathrm{S} 1$ (mata).

${ }^{44}$ Cuando no indicamos nada se trata de mata de manzana: F 2 (mata mansano), F 4 a (id.), F 6 (mata), F 8 (id.), Z 1 (mata de mansano), Z 1c, Z 2 (mata mansano), Z 4 (mata), Tá 1 (íd.), T 1 (id.), A 2, P 2 (mata), B 3 (id.), Co 1, G 1 (mata), G 2 (id.), G 3 (id.), IM 1, Mo 2, Bo 1 (mata), Ca 1 a, b, c, Cr 1 d, e, M 1 a, b, c, M 2 (mata), Mo 1 (id.), N 1 b (id.), S 1 (id.). 
ral' (II, 327) ${ }^{45}$, mata de coco 'cocotero' (II, 328) ${ }^{46}$, mata ciruela, mata de ciruela 'ciruelo' (II, 330) ${ }^{47}$.

Mata + nombre de fruto para designar al árbol que da ese fruto no es recogido por el DRAE-01 ni por Tejera 1993. Núñez y Pérez 1998 dicen de mata que es «planta o arbusto de cualquier tamaño». Es un significado general de la voz. La definición de López Morales 2010 no viene bien con nuestro ejemplo: «planta, excepto árboles grandes».

35. Concha 'mondadura de la patata' (II, 333) ${ }^{48}$.

El DRAE-01 con localización en Colombia y Venezuela indica que es «cáscara, corteza o cubierta exterior». Con este mismo sentido aparece en Tejera 1993 y Núñez y Pérez 1998. López Morales 2010 lo registra para Ho, Ni, Co: N, Ve.

36. Patilla 'sandía' (II, 334) ${ }^{49}$.

El DRAE-01 lo localiza en Colombia, Puerto Rico, República Dominicana y Venezuela. López Morales 2010 lo sitúa en RD, PR, Co, Ve. También lo registran Tejera 1993 y Núñez y Pérez 1998.

37. Concha ‘corteza de árbol' (II, 339) ${ }^{50}$.

El DRAE-01 con localización en Colombia y Venezuela dice esto mismo: «corteza de los árboles». López Morales 2010 lo da para Ho, Co: N, Ve. También en Tejera 1993 y Núñez y Pérez 1998.

38. Floriar 'florecer' (II, 340) $)^{51}$.

El DRAE-01 recoge florear en este sentido con localización en América. López Morales 2010 registra florear(se) en Mx, Gu, Ho, ES, Ni, CR, Pa, Cu,

${ }^{45}$ Cuando no indicamos nada se trata de mata de pera o mata pera: F 2, F 4, F 6 (mata), F 8 (id.), Z 1, Z 2, Z 4 (mata), Tá 1 (mata), T 1 (id.), A 2, A 3, P 2 (mata), Co 1, G 1 (mata), G 2, G 3, An 1, Mo 2, Bo 1 (mata), Ca 1 a, b, c, Cr 1d, e, M 1 a, b, c, M 2, Mo 1 (mata), N 1 b (id.), S 1 (id.).

${ }^{46}$ F 1, F 2, F 4 b, F 5, F 6, F 8, F 9, Z 1, Z 2, Z 4, T 1, Tá 1, P 2 (mata), B 2, B 3, B 4 (mata), A 3, Cr 1, G 1 (mata), G 2, IM 1, Bo 1 (mata), Ca 1b, c, Co 1 (mata), M 1 a, b, M 2, Mo 1 (mata), N 1 a, S 1 (mata).

${ }^{47}$ F 2, F 4, F 5 (mata), F 6 (id.), F 8 (id.), F 9 (id.), Z 1, Z 2, Z 4 (mata), B 1, A 2, A 3, Co 1, Cr 1, G 2 (mata), IM 1, An 1, Mo 2, Ca 1 a (mata de ciruelo), Ca 1b, Ca 1c, Cr 1d, Cr 1 e, M 1 a (mata de cirigüelo), Mo 1 (mata), $\mathrm{N} 1 \mathrm{a}, \mathrm{b}$ (id.), S 1 (id.).

${ }^{48} \mathrm{Fl} \mathrm{1,} \mathrm{F} \mathrm{1,} \mathrm{F} \mathrm{2,} \mathrm{F} \mathrm{3,} \mathrm{F} \mathrm{4,} \mathrm{F} \mathrm{5,} \mathrm{F} \mathrm{6,} \mathrm{F} \mathrm{7,} \mathrm{F} \mathrm{8,} \mathrm{F} \mathrm{9,} \mathrm{F} \mathrm{10,} \mathrm{Z} \mathrm{1,} \mathrm{Z} \mathrm{2,} \mathrm{Z} \mathrm{4,} \mathrm{M} \mathrm{3,} \mathrm{Tá} \mathrm{1,} \mathrm{Tá} \mathrm{2,} \mathrm{T} \mathrm{1,} \mathrm{B} \mathrm{1,}$ B 2, B 3, B 4, A 2, A 3, A 4, L 1, L 2, P 1, P 2, Co 1, Cr 1, G 1, G 2, G 3, G 4, IM 1, An 1, An 2, Mo 2, Bo 1, Ca 1b, c, M 1a, b, c, d, M 2, Mo 1, N 1a, b, S 1, S 3.

${ }^{49}$ Fl 1, F 1, F 2, F 3, F 4, F 4b, F 5, F 6, F 7, F 8, F 9, F 10, Z 1, Z 2, Z 4, Tá 1, Tá 2, M 3, T 1, B 1, B 2, B 3, B 4, A 2, A 3, A 4, L 1, L 2, P 1, P 2, Co 1, Cr 1, G 1, G 2, G 3, G 4, An 1, An 2, S 2, Mo 2, Bo 1, Ca 1a, Ca 1b, c, M 1a, b, c, M 1d, M 2, Mo 1, N 1 a, b, S 1, S 3.

${ }^{50} \mathrm{Fl} \mathrm{1,} \mathrm{F} \mathrm{1,} \mathrm{F} \mathrm{3,} \mathrm{F} \mathrm{4,} \mathrm{F} \mathrm{5,} \mathrm{F} \mathrm{6,} \mathrm{F} \mathrm{7,} \mathrm{F} \mathrm{8,} \mathrm{F} \mathrm{9,} \mathrm{F} \mathrm{10,} \mathrm{Z} \mathrm{1,} \mathrm{Z} \mathrm{2,} \mathrm{Z} \mathrm{4,} \mathrm{M} \mathrm{3,} \mathrm{Tá} \mathrm{1,} \mathrm{L} \mathrm{1,} \mathrm{T} \mathrm{1,} \mathrm{B} \mathrm{1,} \mathrm{B} \mathrm{2,} \mathrm{B} \mathrm{3,}$ B 4, A 2, A 3, A 4, P 1, P 2, Co 1, G 1, G 2, G 3, G 4, IM 1, An 1, An 2, Mo 2, Bo 1, Ca 1a, b, c, M 1a, b, M 2, Mo 1, N 1a, b, S 1, S 3.

${ }^{51}$ Fl 1, F 2, F 3, F 8, Z 1, Z 2, Z 4, M 3, T 1, A 2, A 3, A 4, L 1, L 2, P 1, Co 1, B 2, B 4, Cr 1, G 1, An 1, An 2, S 2, Bo 1, Ca 1a, b, c (florear), M 1a, b, Mo 1, N 1a, S 3. 
RD, Co, Ve, Ec, Bo, Ch, Ur. También florear en Tejera 1993. Núñez y Pérez 1998 registran florear, florar y floriar.

39. Cacuro 'avispa' (II, 353) ${ }^{52}$ en el Estado de Falcón.

La voz no es recogida por el DRAE-01. López Morales 2010 ofrece cacuro y cacure para el oeste de Ve. Tejera 1993 la localiza en los Estados de Falcón y Lara. Núñez y Pérez 1998 la definen como «especie de avispa».

40. Bachaco 'hormiga de alas' (II, 354) ${ }^{53}$.

El DRAE-01 con localización en Venezuela indica que bachaco es «hormiga grande y voraz de los Formicidos, de color rojizo y a veces negro según la especie». López Morales 2010 registra el término en Co y Ve. También con este sentido aparece en Tejera 1993 y Núñez y Pérez 1998.

41. Cocuyo 'insecto fosforescente volador' (II, 356) ${ }^{54}$ e 'insecto fosforescente terrestre' (II, 357) ${ }^{55}$.

El DRAE-01 dice de cocuyo, «insecto coleóptero de América tropical, de unos tres centímetros de longitud, oblongo, pardo y con dos manchas amarillentas a los lados del tórax, por las cuales despide de noche una luz azulada bastante viva». López Morales 2010, como «insecto volador [...] [que] despide de noche una luz azulada bastante viva», lo ofrece para Mx, Ho, $\mathrm{Ni}, \mathrm{Pa}, \mathrm{Cu}, \mathrm{RD}, \mathrm{PR}, \mathrm{Co}, \mathrm{Ve}, \mathrm{Ec}, \mathrm{Bo}, \mathrm{Ar}$ : NO, O. También lo recogen Tejera 1993 y Núñez y Pérez 1998.

42. Jején 'jején (mosquita pequeña que pica)' (II, 359 ${ }^{56}$.

El DRAE-01 ofrece el término con la siguiente explicación: «insecto díptero, más pequeño que el mosquito y de picadura más irritante. Abunda en las playas del mar de las Antillas y en otras regiones de América». López Morales 2010 lo ofrece para Mx, Gu, Ho, ES, Ni, CR, Pa, Cu, RD, Co, Ve, Ec, Pe, Bo, Ch, Py, Ar, Ur. También aparece en Tejera 1993 y Núñez y Pérez 1998.

${ }_{52} \mathrm{Fl} \mathrm{1,} \mathrm{F} \mathrm{1,} \mathrm{F} \mathrm{2,} \mathrm{F} \mathrm{3,} \mathrm{F} \mathrm{4,} \mathrm{F} \mathrm{5,} \mathrm{F} \mathrm{6,} \mathrm{F} \mathrm{7,} \mathrm{F} \mathrm{8,} \mathrm{F} \mathrm{9,} \mathrm{F} 10$.

${ }_{53}$ F 1, F 4b, F 7, Z 1, Z 4, Tá 1 (machaco), L 2, B 1 (hormiga bachaquera), A 1, A 2, Cr 1, An 1, An 2, S 2, Mo 2, Bo 1, Bo 2, Cr 1d (machaco), M 1b (bachaca), M 2 (guachaco), M 3a, b (bachaco culón), Mo 1 (machaco culón), N 1b (machaco), S 3.

${ }_{54}$ F 2, F 5, F 6, F 7 (cocuyito), F 8, F 9, F 10, Z 1, Z 2, M 3, Tá 1, A 1, A 2, A 3, L 2, P1 (cucuí), P2, Co 1, B 4 (cucuí), A 4 (íd.), G 2, G 4 (cucuí), IM 1, An 2, F 4a, M 1a, b (cucuí), Mo 1, N 1a, S 1 , Tá 2.

${ }_{55} \mathrm{Fl} \mathrm{1,} \mathrm{F} \mathrm{1,} \mathrm{F} \mathrm{2,} \mathrm{F} \mathrm{3,} \mathrm{F} \mathrm{4,} \mathrm{F} \mathrm{4b,} \mathrm{F} \mathrm{5,} \mathrm{F} \mathrm{6,} \mathrm{F} \mathrm{10,} \mathrm{Z} \mathrm{1,} \mathrm{Z3,} \mathrm{Z} \mathrm{4,} \mathrm{L} 1$ (cucuí), B 1, B 2, B 3, B 4 (cucuí), A 4 (cocuí), G 1 (id.), G 2 (cucuí), G 3 (id.), G 4 (id.), S 2, An 1, A 2, Bo 1, Bo 2 (cocuí), Ca 1a, b, c (id.), Cr 1b, c, e, Cr 1 d, L 2, M 1a, (cucuí), M 1b (cocuí), M 1c, M 1d, M 2, Mo 1, S 1, S 3 .

${ }_{56} \mathrm{Fl} \mathrm{1,} \mathrm{F} \mathrm{1,} \mathrm{F} \mathrm{2,} \mathrm{F} \mathrm{3,} \mathrm{F} \mathrm{4,} \mathrm{F} \mathrm{5,} \mathrm{F} \mathrm{6,} \mathrm{F} \mathrm{7,} \mathrm{F} \mathrm{8,} \mathrm{F} \mathrm{9,} \mathrm{F} \mathrm{10,} \mathrm{Z} \mathrm{1,} \mathrm{Z} \mathrm{2,} \mathrm{Z} \mathrm{4,} \mathrm{M} \mathrm{3,} \mathrm{L} \mathrm{1,} \mathrm{L} \mathrm{2,} \mathrm{T} \mathrm{1,} \mathrm{B} \mathrm{1,} \mathrm{B} 2$, B 3, B 4, A 2, A 3, P 1, P 2, Co 1, Cr 1, G 1, G 2, G 3, G 4, IM 1, An 1, An 2, Bo 1, Ca 1 a, b, c, M 1b, c, d, M 2, N 1 a, b, Tá 2. 
43. Zancudo 'mosquito zancudo' (II, 360 ${ }^{57}$.

El DRAE-01 registra zancudo para América como «mosquito». López Morales 2010 da la siguiente zona: EU, Mx, Gu, Ho, ES, Ni, CR, Pa, Cu, RD, PR, $\mathrm{Co}, \mathrm{Ve}, \mathrm{Ec}, \mathrm{Pe}, \mathrm{Bo}, \mathrm{Ch}, \mathrm{Ar}$ y Ur, en este último país poco usado. También aparece en Tejera 1993.

44. Coquito 'mariquita' (II, 365) ${ }^{58}$.

No es recogido por el DRAE-01 ni por Tejera 1993. Tampoco figura en López Morales 2010. Núñez y Pérez 1998 dicen que es «nombre común a cualquier especie de coleóptero de pequeñas dimensiones».

45. Chupaflor 'colibrí' (II, 366) $)^{59}$.

El DRAE-01 lo localiza en Colombia, Honduras, México, Puerto Rico y Venezuela. López Morales 2010 lo ofrece para la siguiente área: Mx, Gu, Ho, PR, Co, Ve, Ec, en este último poco usado. No aparece ni en Tejera 1993 ni en Núñez y Pérez 1998.

46. Samuro 'gallinazo' (II, 369) ${ }^{60}$.

El DRAE-01 recoge samuro con localización en Colombia como «zopilote [ave rapaz]» y zamuro para Colombia, Honduras y Venezuela con el mismo significado. López Morales 2010 trae samuro para Co: E y Ve; y zamuro para Ni, Co: E, C, NE y Ve. También en Tejera 1993 y Núñez y Pérez 1998.

47. Padrote 'garañón, asno semental' (II, 388) ${ }^{61}$, 'cerdo semental' (II, $389)^{62}$, 'carnero padre' (II, 390 $)^{63}$, 'boque [macho de la cabra]' (II, 391) ${ }^{64}$.

El DRAE-01 recoge padrote con localización en América Central, Colombia, Ecuador, Puerto Rico, República Dominicana y Venezuela como «macho

${ }_{57} \mathrm{Fl} \mathrm{1,} \mathrm{F} \mathrm{1,} \mathrm{F} \mathrm{2,} \mathrm{F} \mathrm{3,} \mathrm{F} \mathrm{4,} \mathrm{F} \mathrm{4b,} \mathrm{F} \mathrm{5,} \mathrm{F} \mathrm{6,} \mathrm{F} \mathrm{7,} \mathrm{F} \mathrm{8,} \mathrm{F} \mathrm{10,} \mathrm{Z} \mathrm{1,} \mathrm{Z} \mathrm{2,} \mathrm{Z} \mathrm{4,} \mathrm{Tá} \mathrm{1,} \mathrm{L} \mathrm{1,} \mathrm{L} \mathrm{2,} \mathrm{T} \mathrm{1,} \mathrm{A} \mathrm{1,}$ A 2, A 3, P 1, P 2, B 2, B 3, B 4, Cr 1, G 1, G 2, G 3, G 4, An 2, Bo 1, Bo 2, Ca 1a, b, c, Co 1, Cr 1d, Cr 1e, M 1a, b, c, d, M 2, M 3a, b, Mo 1, N 1a, N 1 b, S 1.

${ }_{58}$ F 1, F 2, F 3, F 4 b, F 6, F 7, Z 1, Z 2, M 3, L 1, L 2, P 2, Co 1, B 2, B 4, Cr 1, G 1, S 2, Mo 2 , An 2, Bo 1, Bo 2 (coquito de agua), A 2, Ca 1b, c, M 1d, M 2, S 3, Tá 2.

${ }_{59} \mathrm{Fl} \mathrm{1,} \mathrm{F} \mathrm{2,} \mathrm{F} \mathrm{3,} \mathrm{F} \mathrm{4,} \mathrm{Z} \mathrm{1,} \mathrm{Z} \mathrm{2,} \mathrm{Z} \mathrm{4,} \mathrm{B} 1$ (chupa), A 1, A 2, B 2, B 3, B 4, G 1, IM 1, Bo 2, Ca 1b, Cr 1d, Cr 1e, F 4b, L 1, L 2, M 1a, b, c, d, M 2 (chupas), M 3a, b, Tá 2.

${ }^{60} \mathrm{Fl} \mathrm{1,} \mathrm{F} \mathrm{1,} \mathrm{F} \mathrm{2,} \mathrm{F} \mathrm{3,} \mathrm{F} \mathrm{4,} \mathrm{F} \mathrm{5,} \mathrm{F} \mathrm{6,} \mathrm{F} \mathrm{7,} \mathrm{F} \mathrm{8,} \mathrm{F} \mathrm{9,} \mathrm{F} \mathrm{10,} \mathrm{Z} \mathrm{1,} \mathrm{Z} \mathrm{2,} \mathrm{Z} \mathrm{4,} \mathrm{M} \mathrm{3,} \mathrm{Tá} \mathrm{1,} \mathrm{L} \mathrm{1,} \mathrm{L} \mathrm{2,} \mathrm{T} \mathrm{1,}$ B 1, B 2, B 3, B 4, A 2, A 3, A 4, P 1, P 2, Co 1, Cr 1, G 1, G 2, G 3, G 4, IM 1, An 1, An 2, Mo 2, Bo 1, Ca 1 a, b, c, Cr 1 d, e, M 1 a, b, c, d, M 2, Mo 1, N 1 a, b, S 1, S 3, Tá 2.

${ }^{61}$ F 1, F 4, F 5, F 6, F 10, Z 1, Z 2, Tá 1, L 1, L 2, B 1, B 2, B 4, A 1, A 2, A 3, A 4, P 1, P 2, Co 1 (burro padrote), Cr 1, G 2, G 4, S 2, An 2, Mo 2, Bo 2, Ca 1a, b, c, M la, b, c, d, M 2, N 1a, b, S 1, S 3, Tá 2 .

${ }^{62} \mathrm{Fl} \mathrm{1,} \mathrm{F} \mathrm{1,} \mathrm{F} \mathrm{2,} \mathrm{F} \mathrm{3,} \mathrm{F} \mathrm{4b,} \mathrm{F} \mathrm{5,} \mathrm{F} \mathrm{6,} \mathrm{F} \mathrm{8,} \mathrm{F} \mathrm{9,} \mathrm{F} \mathrm{10,} \mathrm{M} \mathrm{3,} \mathrm{Tá} \mathrm{1,} \mathrm{Z} \mathrm{2,} \mathrm{T} \mathrm{1,} \mathrm{A} \mathrm{1,} \mathrm{A} \mathrm{2,} \mathrm{A} \mathrm{3,} \mathrm{A} \mathrm{4,} \mathrm{P} \mathrm{1,}$ P 2, Co 1, Cr 1, G 2, G 4, S 2, An 1, Mo 2, Bo 1, Bo 2, B 2, Ca 1a, b, c, L 1, L 2, M la, b, c, d, M 2, N 1 a, b, S 1 (puerco padrote), Tá 2.

${ }^{63}$ F 1, F 3, F 4b, F 5, F 6, F 10, M 3, Tá 1, P 1, A 1, A 2, A 3, A 4, Co 1, G 4, Mo 2, B 2, Ca 1 a, b, c, L 2, M 1 a, b, Tá 2 .

${ }^{64}$ Z 2, M 3, Tá 1, A 1, A 2, A 3, Co 1, B 2, Ca 1b, c, F 4b, L 2, M 1 a, b, N 1 a, Tá 2. 
destinado en el ganado para la generación y procreación». López Morales 2010 da la siguiente zona: Gu, Ni, CR, Pa, RD, PR, Co, Ve. También en Tejera 1993 y Núñez y Pérez 1998.

48. Ovejo 'carnero padre' (II, 390) ${ }^{65}$.

El DRAE-01 no recoge la voz. López Morales 2010 la sitúa en Cu, RD, PR, Co, Ve; y Bo: E, S, como rural. Tejera 1993 la localiza en la Región Occidental (incluye los Estados de Zulia, Yaracuy, Falcón y Lara) y Los Andes (incluye los Estados Táchira, Mérida y Trujillo). También en Núñez y Pérez 1998.

49. Cachos 'cuernos' (II, 445) ${ }^{66}$.

El DRAE-01 con localización en América indica que cacho es «cuerno (de animal)». López Morales 2010 da el área de Mx: SE, Gu, Ho, ES, Ni, CR, Pa, RD, Co, Ve, Ec, Pe, Bo, Ch, Ar: O. También en Tejera 1993 y Núñez y Pérez 1998.

50. Trompa ${ }^{67}$ 'hocico del cerdo' (II, 450) ${ }^{68}$ y 'morro del caballo' (II, $436)^{69}$.

El DRAE-01 indica «hocico del cerdo» para Argentina y Uruguay. Tejera 1993 da la siguiente explicación: "parte saliente apuntada de la cara de algunos mamíferos, donde tienen la nariz y la boca. Hocico». López Morales 2010 lo recoge como «hocico del cerdo» en Mx, Ho, ES, Ni, RD, PR, Ar, Ur. No incluye Venezuela. Y como «hocico de los mamíferos» para Ni y Co. Núñez y Pérez 1998 no lo traen.

\section{Conclusiones}

Hemos analizado un total de 50 voces usuales en Venezuela que el hablante del español peninsular no conoce. De estas, el DRAE-01 no recoge 11 . Resulta que solo un $22 \%$ de los términos estudiados no son registrados por la Academia. Las voces que faltan en el DRAE-01 son las siguientes:

capino 'albino' [localizado en Ve por López Morales 2010]

picar 'guiñar el ojo’ [en RD y Ve según López Morales 2010]

mocho 'cojo'

${ }^{65} \mathrm{Fl} \mathrm{1,} \mathrm{F} \mathrm{2,} \mathrm{F} \mathrm{8,} \mathrm{F} \mathrm{9,} \mathrm{Z} \mathrm{1,} \mathrm{Z} \mathrm{3,} \mathrm{Z} \mathrm{4,} \mathrm{P} \mathrm{2,} \mathrm{B} \mathrm{4,} \mathrm{Cr} \mathrm{1,} \mathrm{G} \mathrm{1,} \mathrm{G} \mathrm{2,} \mathrm{G} \mathrm{3,} \mathrm{IM} \mathrm{1,} \mathrm{S} \mathrm{2,} \mathrm{An} \mathrm{2,} \mathrm{M} \mathrm{1c,} \mathrm{d,} \mathrm{M} \mathrm{2,}$ $\mathrm{S} 1, \mathrm{~S} 3$.

${ }_{66}^{6} \mathrm{Fl} \mathrm{1,} \mathrm{F} \mathrm{1,} \mathrm{F} \mathrm{2,} \mathrm{F} \mathrm{3,} \mathrm{F} \mathrm{4,} \mathrm{F} \mathrm{5,} \mathrm{F} \mathrm{6,} \mathrm{F} \mathrm{7,} \mathrm{F} \mathrm{8,} \mathrm{F} \mathrm{9,} \mathrm{F} \mathrm{10,} \mathrm{Z} \mathrm{1,} \mathrm{Z} \mathrm{2,} \mathrm{Z} \mathrm{4,} \mathrm{M} \mathrm{3,} \mathrm{L} \mathrm{1,} \mathrm{L} \mathrm{2,} \mathrm{T} \mathrm{1,} \mathrm{B} \mathrm{1,} \mathrm{B} \mathrm{2,}$ B 4, A 2, A 3, A 4, P 1, P 2, Co 1, Cr 1, G 1, G 2, G 3, IM 1, An 1, An 2, S 2, Mo 1, Mo 2, Bo 1, Bo 2, Ca 1a, b, c, M 1a, b, c, d, M 2, N 1a, b, S 1, S 3, Tá 2.

${ }_{67}$ El número redondo de 50 formas no es meditado. Es puramente fortuito.

${ }^{68}$ Fl 1, F 1, F 2, F 4, F5, F 6, F 7, F 10, Tá 1, Z 2, T 1, P 2, B 1, B 4, Cr 1, G 1, G 2, G 3, G 4, IM 1, S 2, Mo 2, An 2, Bo 1, Bo 2, Ca 1a, b, M 1a, b, d, M 2, Mo 1, S 3, Tá 2.

${ }^{69}$ F 8, Z 1, Z 2, Tá 1, B 1, B 4, P 2, Cr 1, G 1, G 4, An 1, Mo 2, Bo 1, Bo 2, B 2, G 3, M 1 a, b, M 2, Mo 1, N 1 a, b, S 1 . 
braga 'mono (buzo, overol)' [en Ve según López Morales 2010]

cintillo ‘cinta para el pelo' [en Mx, Ho, Ni, Cu, Co: N, Ve, Pe, Bo, Ch, Py, según López Morales 2010]

pasador 'cerrojo'

a chuco, en chuco 'llevar a hombros', 'llevar a cuestas'

kinder 'enseñanza anterior a la primaria' [en EU, Mx, Gu, Ho, Pa, RD, PR, Co, Ve, Ec, Bo, Ch, Ar, según López Morales 2010]

mata + nombre del fruto 'nombre del árbol'

cacuro 'avispa' [oeste de Ve según López Morales 2010]

coquito 'mariquita'

Hay voces recogidas en el DRAE-01 pero falta la localización de Venezuela. De esta manera tenemos:

maneto 'estevado' que el diccionario académico trae para Honduras y Nicaragua [López Morales lo localiza en Ve y Co pero en este último país como poco usado]

trompa 'hocico del cerdo' que el DRAE-01 da para Argentina y Uruguay [López Morales 2010 lo recoge como «hocico del cerdo» en Mx, Ho, ES, Ni, RD, PR, Ar, Ur. No incluye Venezuela]

La mayoría de los términos estudiados por nosotros incluidos en Alvar 2001a son recogidos por el DRAE-01 con la marca de «Venezuela», lo cual da idea de la diligencia que ha tenido la Academia (o los venezolanos) por incluir voces de este país. De esta manera, tenemos:

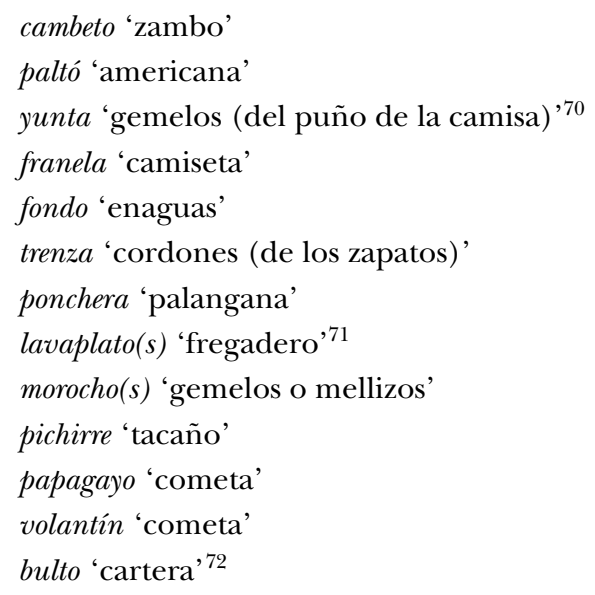

70 También el DRAE-01 lo da para Puerto Rico.

71 También para Chile y Colombia.

72 También la Academia lo localiza en Costa Rica. 


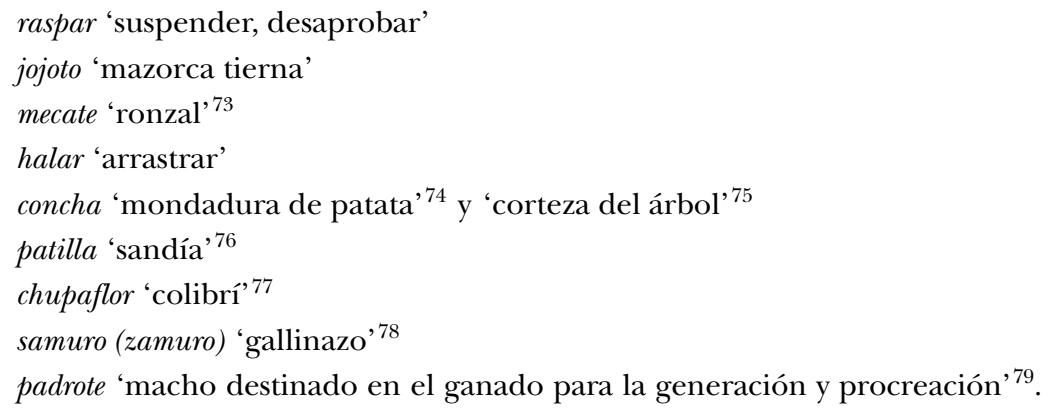

Sabemos que Venezuela cuenta con una buena lexicografía, unos excelentes diccionarios de venezolanismos tales como Tejera 1993 o Núñez y Pérez 1998, que registran muy detalladamente el léxico venezolano.

Siempre hemos trabajado con los atlas y en ellos hemos encontrado muchas palabras que no estaban recogidas por los diccionarios y nos hemos referido a la aportación de voces de la geografía lingüística a la lexicografía, pero en esta ocasión hemos acudido al Atlas de Venezuela y nos hemos encontrado con un porcentaje alto, el $78 \%$, de las voces que están incluidas en el DRAE-01. Ahora bien, aunque la Academia las localice en Venezuela, la mayoría de ellas se usan en más países americanos, como nos indica el Diccionario de americanismos de la Asociación de Academias de la Lengua Española 2010, con lo cual nos hacemos una idea de la gran cantidad de información que arroja este Diccionario. Esta obra nos ofrece una luz nueva sobre todo el léxico del español de América porque además de enriquecer la localización de las voces con muchos más países, nos da el área que ocupa cada término. El Diccionario de americanismos no es un diccionario más del español de América. Es el Diccionario del español de América por excelencia. Estamos muy cerca del léxico americano con esta obra brillante y moderna que la Asociación de Academias de la Lengua Española ha realizado con su Secretario y Director del Diccionario a la cabeza, Humberto López Morales. Con este Diccionario no solo está bien recogido el léxico venezolano sino también el léxico de todo el español de América.

${ }^{73}$ También para América Central y México.

${ }^{74}$ También para Colombia.

${ }^{75}$ También para Colombia.

${ }^{76}$ También para Colombia, Puerto Rico y República Dominicana.

77 También para Colombia, Honduras, México y Puerto Rico.

78 También para Colombia y Honduras.

${ }^{79}$ También para América Central, Colombia, Ecuador, Puerto Rico y República Dominicana. 


\section{APÉNDICE 1.}

\section{Puntos De Encuesta}

Estado
APURE
Localidades:
A 1 = Palmarito
A 2 = Guasdualito
A 3 = Elorza
A $4=$ Achagua
Estado
ANZUÁTEGUI
Localidades:
An 1 = Puerto de la Cruz
An 2a = El Tigre
An 2b = El Tigre

\section{Estado}

BARINAS

Localidades:

B 1 = Barinas

B 2 = Dolores (distrito de Rojas)

B 3 = Ciudad de Nutrias

B $5 \mathrm{a}=$ Arismendi

B $5 b=$ Arismendi

\section{Estado}

Ciudad Bolíinar

Localidades:

Bo 1 = Upata

Bo 2 = Mariño

\section{Estado}

CARABOBo

Localidades:

Ca 1a = Naguaragua (Valencia)

$\mathrm{Ca} 1 \mathrm{~b}=$ Naguaragua (Valencia)

$\mathrm{Ca} 1 \mathrm{c}=$ Valencia

\section{Estado}

Cojedes

Localidades:

Co $1=$ Tinaquillo

\section{Estado}

\section{CARACAS}

Localidades:

Cr 1a =Caracas

$\mathrm{Cr} 1 \mathrm{~b}=$ Caracas

Cr 1c= Caracas

Cr $1 \mathrm{~d}=$ Caracas

Cr 1e = Caracas

\section{Estado}

\section{FALCÓN}

Localidades:

F 1 = San Juan

F 2 = Pueblo Cumarelo

F 3 = Capataria

$\mathrm{F} 4 \mathrm{a}=$ Urumaco

$\mathrm{F} 4 \mathrm{~b}=$ Urumaco

F 5 = Curimagua

F $6=$ Sta. Cruz del Bucaral

F 7 = Zaragoza

F $8=$ La Cruz de Taratara

F 9 = Churuguara

F $10=$ Mapararí

F 11 = Adícora

\section{Estado}

GUÁRICO

Localidades:

G 1 = El Sombrero

G 2a = Las Mercedes del Llano

$\mathrm{G} 2 \mathrm{~b}=$ Las Mercedes del Llano

G 3 = Valle de la Pascua

G 4 = Alta Gracia de Osorio 


\section{Estado}

IsLA MARGARITA

Localidades:

IM 1 = Porlamar

\section{Estado}

LARA

Localidades:

L 1 = Carora

L 2 = Sanares (Barquisimeto)

\section{Estado}

Monagas

Localidades:

Mo 1 = Aragua de Maturín

Mo 2 = Maturín

\section{Estado}

Portuguesa

Localidades:

P 1 = Acarigua

P 2 = Chabasquén

\section{Estado \\ SUCRE}

Localidades:

S 1 = Río Caribe

S 2 = Carúpano

S 3 = Cumaná

\section{Estado \\ Trujillo \\ Localidades: \\ $\mathrm{T} 1$ = Trujillo}

\section{Estado}

\section{TÁCHIRA}

Localidades:

Tá 1 = San Cristóbal

Tá 2 = Táriba

\section{Estado \\ YARACUY \\ Localidades: \\ Y la = Nirgua \\ $\mathrm{Y} 1 \mathrm{~b}=$ Nirgua}

\section{Estado}

Zulia

Localidades:

Z 1a = Maracaibo

$\mathrm{Z} 1 \mathrm{~b}=$ Maracaibo

$\mathrm{Z} 1 \mathrm{c}=$ Maracaibo

$\mathrm{Z} 2$ = Cajaseca

Z 3 = Encontrados

Z 4 = San Carlos 


\section{APÉNDICE 2.}

\section{ÍNDICE DE VOCES ANALIZADAS}

a chuco 'llevar a cuestas', 'llevar a hombros' $\$ 21$

bachaco 'hormiga de alas' $\$ 40$

braga 'mono (buzo, overol)' $\$ 9$

bulto 'cartera' $\$ 27$

cachos 'cuernos' $\$ 49$

cacuro 'avispa' $\$ 39$

cambeto 'zambo' $\$ 45$

cambur 'plátano, banano (planta)' $\$ 30$

cambural 'platanar (terreno de plátanos)' $\$ 31$

capino 'albino' $\$ 2$

catire 'rubio' $\$ 1$

chupaflor 'colibrí' $\$ 45$

cintillo ‘cinta para el pelo’ $\$ 13$

cobija 'manta' 17

cocuyo 'insecto fosforescente terrestre' 'insecto fosforescente volador' $\$ 41$

concha 'corteza de árbol' §37; 'mondadura de la patata' $\$ 35$

coquito 'mariquita' $\$ 44$

en chuco 'llevar a cuestas' 'llevar a hombros' $\$ 21$

floriar 'florecer' $\$ 38$

flux 'traje [de hombre]' $\$ 7$

fondo 'enaguas' $\$ 12$

franela 'camiseta' \$11

ganchos 'horquillas para el pelo' \$25

halar 'arrastrar' $\$ 33$

jején 'jején (mosquita pequeña que pica)' $\$ 42$

jojoto 'elote (mazorca tierna)' \$29

kinder 'enseñanza anterior a la primaria' \$26

lavaplatos 'fregadero' $\$ 18$

limosnero 'mendigo' \$24 maneto 'estevado' $\$ 6$

mata 'manzano' 'naranjo' \$34

mata de ciruela 'ciruelo' $\$ 34$

mata ciruela 'ciruelo' $\$ 34$

mata de coco 'cocotero' $\$ 34$

mata de higo 'higuera' \$34

mata de limón 'limonero' $\$ 34$

mata de manzana 'manzano' $\$ 34$

mata de manzano 'manzano' $\$ 34$

mata de naranja 'naranjo' $\$ 34$

mata de pera 'peral' $\$ 34$

mata naranjo 'naranjo' $\$ 34$

mecate 'ronzal' \$32

mocho 'cojo' $\$ 4$

morocho(s) 'gemelos o mellizos' \$19

ovejo 'carnero padre' $\$ 48$

padrote 'boque [macho de la cabra]' 'carnero padre', 'cerdo semental',

'garañón, asno semental' $\$ 47$

paltó 'americana' $\$ 8$

papagayo 'cometa' $\$ 22$

pasador 'cerrojo' $\$ 15$

patilla 'sandía'§36

picar 'guiñar el ojo' §3

pichirre 'tacaño' $\$ 20$

ponchera 'palangana' $\$ 16$

raspar 'suspender, desaprobar' \$28

samuro 'gallinazo' $\$ 46$

trenza 'cordones' $\$ 14$

trompa 'hocico del cerdo' 'morro del caballo' $\$ 50$

volantín 'cometa' $\$ 23$

yunta 'gemelos del puño de la camisa' $\$ 10$

zancudo 'mosquito zancudo' $\$ 43$ 
Abreviaturas citadas del Diccionario de americanismos de la Asociación de Academias de la Lengua Española, H. López Morales (dir.) 2010:

$$
\begin{aligned}
& \mathrm{Ar}=\text { Argentina } \\
& \mathrm{Ch}=\text { Chile } \\
& \mathrm{Co}=\text { Colombia } \\
& \mathrm{CR}=\text { Costa Rica } \\
& \mathrm{Cu}=\text { Cuba } \\
& \mathrm{E}=\text { este } \\
& \mathrm{ES}=\text { El Salvador } \\
& \mathrm{EU}=\text { Estados Unidos } \\
& \mathrm{Gu}=\text { Guatemala } \\
& \mathrm{Ho}=\text { Honduras } \\
& \mathrm{Mx}=\text { México } \\
& \mathrm{N}=\text { norte } \\
& \mathrm{NE}=\text { noreste }
\end{aligned}
$$

$$
\begin{aligned}
& \mathrm{Ni}=\text { Nicaragua } \\
& \mathrm{NO}=\text { noroeste } \\
& \mathrm{Pa}=\text { Panamá } \\
& \mathrm{Pe}=\text { Perú } \\
& \mathrm{PR}=\text { Puerto Rico } \\
& \mathrm{Py}=\text { Paraguay } \\
& \mathrm{RD}=\text { República Dominicana } \\
& \mathrm{S}=\text { sur } \\
& \mathrm{SE}=\text { sureste } \\
& \mathrm{SO}=\text { suroeste } \\
& \mathrm{Ur}=\text { Uruguay } \\
& \mathrm{Ve}=\text { Venezuela }
\end{aligned}
$$

\section{REFERENCIAS BIBLIOGRÁFICAS}

ACAdemia EsPañola (1726): Diccionario de autoridades, Madrid, Reproducción facsímil de 1976, Madrid, Gredos.

- (1861): Reglamento de la Real Academia Española, Madrid.

- (1878): Reglas para la corrección y aumento del diccionario vulgar, Madrid.

- (1925): Diccionario de la lengua española, 15 $5^{\underline{a}}$ ed., Madrid.

- (2001): Diccionario de la lengua española, Madrid, Espasa.

Alvar, M. (1982): «Atlas lingüísticos y diccionarios», Lingüística Española Actual 4, pp. 253-323.

- (2000a): El español en el sur de los Estados Unidos. Estudios, encuestas, textos, Alcalá de Henares, Universidad de Alcalá y La Goleta Ediciones.

- (2000b): El español en la República Dominicana. Estudios, encuestas, textos, edición al cuidado de Antonio Alvar Ezquerra, Alcalá de Henares, Universidad de Alcalá y La Goleta Ediciones.

- (2001a): El español en Venezuela. Estudios, mapas, textos, edición al cuidado de Antonio Alvar y Florentino Paredes, Alcalá de Henares, Universidad de Alcalá, La Goleta Ediciones y Agencia Española de Cooperación Internacional.

- (2001b): El español en Paraguay. Estudios, encuestas, textos, edición al cuidado de Antonio Alvar Ezquerra, Jairo García y José Ramón Franco, Alcalá de Henares, Agencia Española de Cooperación Internacional, Universidad de Alcalá y La Goleta Ediciones. 
Asociación de Academias de la lengua Española y López Morales, H. (dir.) (2010): Diccionario de americanismos, Lima, Santillana.

Navarro Carrasco, A. I. (1988): Estructuras del léxico meridional según el ALEA, Málaga, Universidad de Málaga.

- (1990): «Voces del Atlas lingüistico y etnográfico de Andalucía no recogidas por el Diccionario académico (1984), 20aㅡ ed.», Español Actual 54, pp. 41-90.

- (1992): "Términos del tomo I del ALEICan que no figuran en el Diccionario de la Real Academia Española (1984), 20登 ed.», en Ariza, M., Cano, R., Mendoza, J. Ma . y Narbona, A. (eds.), Actas del II Congreso Internacional de Historia de la Lengua Española, Madrid, Pabellón de España, pp. 1251-1265.

- (1993): «Geografía lingüística y diccionarios», Estudios de Lingüística de la Universidad de Alicante 9, pp. 73-96.

- (1996): El Atlas de Canarias y el Diccionario académico, Alicante, Universidad de Alicante.

- (1998): Comentario de mapas lingüisticos españoles, Alicante, Universidad de Alicante.

NúÑez, R. y PÉrez, F. J. (1998): Diccionario del habla actual de Venezuela. Venezolanismos, voces indigenas, nuevas acepciones, Caracas, Universidad Católica Andrés Bello.

Tejera, M. J. (dir.) (1993): Diccionario de venezolanismos, Caracas, Universidad Central de Venezuela, Academia Venezolana de la Lengua y fundación Edmundo e Hilde Schnoegass. 
